\title{
Different inhibitory control components predict different levels of language control in bilinguals
}

\author{
Shuhua $\mathrm{Li}^{1,2} \cdot$ Mona Roxana Botezatu ${ }^{3}$ - Man Zhang ${ }^{1}$ - Taomei Guo ${ }^{1,4}$
}

Accepted: 17 December 2020 / Published online: 4 January 2021

(C) The Psychonomic Society, Inc. 2021

\begin{abstract}
In recent years, some studies have started to explore the impact of individual general executive functions (EFs) on bilingual language control. To our knowledge, few studies have systematically examined various components of EFs on different levels of language control in bilinguals. In two experiments, we investigated the effects of two components of IC on different levels of bilingual language control. The language-switching task was used to tap into language control at different levels. The Simon task was used to measure interference suppression in Experiment 1, and a go/no-go task was used to measure response inhibition in Experiment 2. Experiment 1 found that the smaller the Simon effect was, the larger the asymmetry of switch costs was. Experiment 2 found that the shorter the go response time was, the larger the global slowing effect was. Taken together, these findings suggest that the interference suppression component of domain-general IC facilitates local level language control, while response inhibition impacts global level language control in bilinguals.
\end{abstract}

Keywords Language control · Interference suppression · Response inhibition · Bilingualism

\section{Introduction}

When speaking one language, bilinguals are subject to competition and interference from the other language because both languages are activated in parallel (e.g., Costa \& Caramazza, 1999; Guo \& Peng, 2006; Mishra \& Singh, 2016). An important question is how they select words from the target language while avoiding interference of the non-target language. According to the inhibitory control (IC) model (Green, 1998), bilinguals need to recruit IC for lexical selection among two languages.

The language-switching task, which requires participants to name pictures or digits in different languages according to

Taomei Guo

guotm@bnu.edu.cn

1 State Key Laboratory of Cognitive Neuroscience and Learning \& IDG/McGovern Institute for Brain Research, Beijing Normal University, Beijing 10085, People's Republic of China

2 School of Psychology, Shanghai University of Sport, Shanghai 200438, People's Republic of China

3 Department of Speech, Language and Hearing Sciences, University of Missouri, Columbia, MO 65211, USA

4 Center for Collaboration and Innovation in Brain and Learning Sciences, Beijing Normal University, Beijing 10085, People's Republic of China certain cues (e.g., Christoffels, Firk, \& Schiller, 2007; Costa \& Santesteban, 2004; Meuter \& Allport, 1999), is a typical paradigm for investigating language control mechanisms. When the language used to name the current stimulus is different from that used to name a previous stimulus, it is termed a switching condition. When the language used for naming the current stimulus is the same as that for the previous stimulus, it is called a non-switching condition. The difference in response times (RTs) or accuracy rates between these two conditions is referred to as the switch cost.

Many studies have shown that switching into the L1 induces a greater switch cost than switching into the L2 (i.e., asymmetry of switch costs; e.g., Costa \& Santesteban, 2004; Ma, Li, \& Guo, 2016; Meuter \& Allport, 1999; Philipp, Gade, \& Koch, 2007, Experiment 1). Another main finding is that longer RTs are induced by naming in L1 than in L2 under mixed naming contexts (e.g., Costa \& Santesteban, 2004; Fu et al., 2017; Gollan \& Ferreira, 2009; Gollan, Kleinman, \& Wierenga, 2014). This is known as the reversed language dominance effect. It has been claimed that the asymmetry of switch costs represents reactive language control at the local (trial-by-trial) level and stronger reactive inhibition of L1 (e.g., Christoffels et al., 2007; Ma et al., 2016; Wu, Kang, Ma, Gao, \& Guo, 2018; for a review, see Declerck, 2020). In contrast, the reversed language dominance effect may reflect proactive language control at a global (block-by-block) 
level under mixed naming contexts, with stronger proactive inhibition of L1 (e.g., Bobb \& Wodniecka, 2013; Kroll, Bobb, \& Wodniecka, 2006; Kleinman \& Gollan, 2018; Wu et al., 2018; for a review, see Declerck, 2020).

However, neither the asymmetry of switch costs, nor the reversed language dominance effect have been replicated in all studies on bilingual language production. Specifically, the asymmetry of switch costs may be modulated by language context (voluntary switching, e.g., Gollan \& Ferreira, 2009), preparation time (e.g., Verhoef, Roelofs, \& Chwilla, 2009), language proficiency (e.g., Costa \& Santesteban, 2004), types of stimuli (e.g., Finkbeiner, Almeida, Janssen, \& Caramazza, 2006), and domain-general IC (e.g., Liu, Rossi, Zhou, \& Chen, 2014). Likewise, the presence of the reversed language dominance effect is also modulated by factors such as relative accessibility of the two languages (e.g., Declerck et al., 2020) and types of stimuli (e.g., Meuter \& Allport, 1999). Overall, these studies suggest that individual variation in linguistic and cognitive abilities may reveal critical clues about the cognitive nature of bilingual language control.

An important question is whether bilingual language control is language-specific or domain-general. Some studies have found that individual differences in IC may have an impact on bilingual language control. For example, Liu et al. (2014) found asymmetrical switching costs in bilinguals with low IC, and symmetric switching costs in those with high IC, indicating that domain-general inhibition has an impact on lexical selection in bilinguals. In another study, Liu, Liang, Dunlap, Fan, and Chen (2016b) examined the effect of IC training on language control. The results showed that bilinguals with low IC achieved symmetrical switching costs after 6 days of task-switching training, indicating that inhibition training can enhance language control efficiency in participants with low IC. Linck, Schwieter, and Sunderman (2012) found that a smaller Simon effect predicted a smaller L1 switching cost in English-French-Spanish trilinguals, and claimed that trilinguals with better IC have more rapid inhibition of the non-target lexical items. In contrast, De Bruin, Roelofs, Dijkstra, and Fitzpatrick (2014) found a negative correlation between the Simon effect and the L1 switching cost, but a positive correlation between the Simon effect and L2/L3 switching costs. However, the authors did not find any correlation between switching costs and stop-signal RTs for the stop-signal task.

Other studies have tried to determine whether bilingual language control is domain-specific or domain-general by examining the relationship between language switching and task switching. Some studies found a correlation between these two types of switching costs (e.g., Declerck, Grainger, Koch, \& Philipp, 2017; Liu, Fan, Rossi, Yao, \& Chen, 2016a; Prior \& Gollan, 2011), while others failed to find a relationship (e.g., Calabria, Hernández, Branzi, \& Costa, 2012; Kang, Ma, Li, Kroll, \& Guo, 2020; Prior \& Gollan,
2013). The diverging results may be caused by the variability in task similarity between language switching and task switching (e.g., Declerck et al., 2017).

It is worth noting that IC includes at least two sub-components: interference suppression and response inhibition (Bunge, Dudukovic, Thomason, Vaidya, \& Gabrieli, 2002). Interference suppression refers to the ability to inhibit irrelevant information and respond correctly, while response inhibition refers to the ability to inhibit responses to dominant behaviors (e.g., Friedman \& Miyake, 2004; Nigg, 2000; Van Boxtel, van der Molen, Jennings, \& Brunia, 2001). The Simon task is one of the classical paradigms for measuring interference suppression, where smaller Simon effects (i.e., difference between incongruent trials and congruent trials) have better index interference suppression ability (e.g., Bialystok, Craik, Klein, \& Viswanathan, 2004; Grady, Luk, Craik, \& Bialystok, 2015; Simon \& Rudell, 1967). The go/no-go task is one of the classical paradigms for measuring response inhibition, where shorter RTs in go trials indicate better response-inhibition ability (e.g., Benikos, Johnstone, \& Roodenrys, 2013; Jodo \& Inoue, 1990; Manuel, Grivel, Bernasconi, Murray, \& Spierer, 2010). Many studies reported a correlation between fast go responses and an increased requirement for inhibition on no-go trials (e.g., Lindqvist \& Thorell, 2009; Manuel et al., 2010; Smith, Johnstone, \& Barry, 2006). From this perspective, previous studies have mainly revealed the relationship between interference suppression and language control in bilinguals, but the results of these studies are far from consistent. In addition to differences in participants and tasks, one possibility is that most studies used the switching cost for a certain language, rather than the asymmetry of switching costs, as the measurement of language control. However, based on the IC model (Green, 1998), switching costs alone cannot represent language control, but the difference between the switch costs in two languages reveals the selective engagement of extra inhibitory mechanisms for the dominant language. Therefore, it is more reasonable to use the asymmetry of switching costs instead of the switching cost of a certain language to represent the process of local language control (Jylkkä, Lehtonen, Lindholm, Kuusakoski, \& Laine, 2017). Among all previous studies, only Liu et al. (2014) used the asymmetry of switching cost as a measure of language control. However, participants in their study were split into highand low-ability groups by medians, which may have led to loss of some information, because interference inhibition, as a continuous predictor, was used as a categorical factor. Plonsky (2011) believes that converting continuous predictors into categorical factors may affect statistical power. Linck et al. (2012) and Jylkkä et al. (2017) adopted more sensitive methods with linear mixed-effects models and evaluated IC ability as a continuous predictor, taking into account differences in subjects and items. In addition, previous studies mainly focused on the impact of interference 
suppression on switching costs, which could not fully explain the relationship between IC and language control. Bialystok, Craik, and Ryan (2006) argued that different components of executive functions entail different processes of language control. For bilinguals, inhibiting the response in the non-target language is possibly similar to how other habitual responses are intentionally withheld by response suppression, while inhibiting the representation of the non-target language that conflicts with the currently active system is possibly similar to inhibiting the irrelevant mental representation in the process of interference inhibition.

To fully explore the impact of IC on language control in bilinguals, the present study aimed to systematically examine how interference suppression and response inhibition - two different components of IC - affect language control at the local and global levels. Two experiments were carried out, in which unbalanced Chinese-English bilinguals were asked to name pictures in an appropriate language according to visually presented cues. Overall, it was predicted that bilinguals would exhibit a switching cost for both languages (i.e., longer naming latencies to switch relative to non-switch trials) and that the switching cost would be asymmetrical: a greater cost associated with switching from the less dominant L2 to the dominant L1. It was further predicted that bilinguals would be overall slower to name in the native language under mixed naming conditions. The asymmetry of switching costs and the reversed language domiance effect were used to measure language control at the local and global levels, respectively. In Experiment 1, participants were also asked to complete the Simon task, which was used to measure bilinguals' interference suppression ability. In Experiment 2, a similar group of bilinguals was selected to complete the go/no-go task, where go RT was used to represent response-inhibition ability. Linear mixed-effects models were used to analyze the influence of interference suppression and response inhibition, respectively, on language control at different levels.

\section{Experiment 1}

\section{Methods}

\section{Participants}

Fifty-four Chinese-English bilinguals (31 females) with an average age of 22.34 years $(S D=2.43)$ participated in Experiment 1 . Sample size was based on availability, so no a priori power analysis was feasible. Therefore, we performed a post hoc power analysis based on Monte Carlo simulation (number of simulation $=1,000$ ) using the SIMR package in $\mathrm{R}$, which is designed to work with linear fixed-effect models that can be fit with lmerTest (see Green, Macleod, \& Nakagawa, 2016, for more details). Specificly, an estimated power of
$80 \%$ and higher is considered to be adequate (Arend \& Schfer, 2018).

All participants were right-handed, with normal or corrected vision. The average age when they began learning English was 10.13 years $(S D=2.31)$. All participants passed the College English Test-Band 4 (CET-4, full score 710, threshold 425 as a passing score) with an average score of $520(S D=47)$. According to their self-rating scores on a 10 point scale, their proficiency in Chinese was significantly higher than that in English, $t(53)=13.82, p<0.001$, indicating that they were unbalanced bilinguals. Based on previous studies (Chen et al., 2019; Wu et al., 2018), we also used the Raven score and O-span accuracy score to make sure that the two participant groups were matched in fluid intelligence and working memory.

Detailed information about participants' linguistic and cognitive abilities is shown in Table 1.

\section{Materials}

The materials were 48 line drawings selected from the picture set of Snodgrass and Vanderwart (1980). Among them, eight line drawings were used as practice or filler trials.

\section{Procedure}

The experiment was approved by the Institutional Review Board of Beijing Normal University. All participants signed an informed consent form before the experiment. All participants completed the cued language-switching task first, followed by the Simon task.

Cued language-switching task Participants were first familiarized with the Chinese and English names of each picture. For each trial, a fixation cross was presented for $300 \mathrm{~ms}$, and followed by a blank screen for $200 \mathrm{~ms}$. Then a picture together

Table 1 Means for individual difference measures

\begin{tabular}{lccl}
\hline & Experiment 1 & Experiment 2 & $p$ \\
\hline Age (years) & 22.34 & 22.85 & 0.236 \\
Age of L2 acquisition (years) & 10.13 & 9.44 & 0.134 \\
CET-4 scores & 520 & 526 & 0.551 \\
L1 self-rating scores & 7.7 & 7.84 & 0.574 \\
L2 self-rating scores & 4.87 & 5.24 & 0.11 \\
O-span accuracy of recall & 84.51 & 82.44 & 0.238 \\
Raven scores & 56 & 56 & 0.303 \\
\hline
\end{tabular}

$L 1=$ first language, $L 2=$ second language, $C E T-4=$ College English Test-Band 4, a compulsory English proficiency test for Chinese college students with a full score of 710 
with a colored frame were presented in the center of the screen. Participants were asked to name each picture as quickly and accurately as possible in the language indicated by the color of the frame. Participants had up to 2,200 ms to respond to each trial. Their responses were audio recorded and scored offline for accuracy by trained independent raters. There were eight blocks in the formal experiment, each including four conditions: L1 switching, L1 non-switching, L2 switching, and L2 non-switching. Each block comprised one filler trial and 40 critical trials. Overall, there were 80 trials for L1 switching, L1 nonswitching, L2 switching, L2 nonswitching, respectively. The ratio of switching trials and nonswitching trials was 1:1. All trials in each block were presented in a pseudo-random sequence, so that no more than two trials of the same condition presented in a row. All pictures appeared once in each block. To make sure each picture was presented in each condition, the 40 critical pictures were randomly divided into four subsets and assigned to different conditions in different blocks with a Latin square design.

Half of the participants were instructed to name pictures in Chinese when the color of the frame was green and in English when the color was orange; the other half were instructed to name pictures in Chinese when the color was orange and in English when the color was green.

Simon task For each trial, a fixation cross was presented for $350 \mathrm{~ms}$, followed by a blank screen for $150 \mathrm{~ms}$. Then, either a red or a blue square was presented against a white background for $2,000 \mathrm{~ms}$, with the same probability of occurrence for each color. The stimulus appeared on the left, center, or right side of a computer screen, with the same probability of occurrence for each position. Participants were required to respond as quickly and accurately as possible according to the color of the square by pressing a key on the right side of the keyboard for red squares and a key on the left side of the keyboard for blue squares. The task comprised three blocks, each consisting of three conditions: congruent (the stimulus appeared in the same position as the key), incongruent (the stimulus appeared in the opposite position of the key), and neutral (the stimulus appeared in the center of the screen). Each block comprised 42 trials. The Simon effect was computed as the difference in correct-response latency between incongruent and congruent conditions, and was used as a measure for interference inhibition in this experiment.

\section{Data analysis}

Analyses were implemented in $\mathrm{R}$ version 3.5.2 ( $\mathrm{R}$ Development Core Team, 2016). Correct-response latencies were analyzed using linear mixed-effects models with random effects of participants and items. Mixed-effects models were fitted using the lme4 package version 1.1-21 (Bates, Maechler,
Bolker, \& Walker, 2014) and the lmerTest package version 3.1-0 (Kuznetsova, Brockhoff, \& Christensen, 2015).

To help reduce collinearity within the model, the Simon effect was centred around the mean value (Cunnings, 2012). In this model, Language (L1, L2), Trial type (switching, nonswitching), and the Simon effect were fixed effects. The model also included random intercepts for subjects and items, and by-subject and by-item random slopes for language, trial type, and their interaction. Significance ( $p$ value) was calculated with Satterthwaite's approximations (Kuznetsova et al., 2015). Dummy coding was performed for language trials $(\mathrm{L} 1=0, \mathrm{~L} 2=1)$ so that $\mathrm{L} 1$ was regarded as the baseline and the Language parameter indicated the size and direction of the difference in RT between L2 and L1 conditions. Contrast coding was performed for trial type trials (non-switching = 0.5 , switching $=0.5$ ) so that the intercept estimated the mean results of the baseline condition across both switching and non-switching conditions, and the Trial type parameter estimated the switching costs' magnitude in the baseline condition. The Language $\times$ Trial type interaction estimated whether there was a difference in switching cost between L1 and L2. The Simon effect parameter estimated the size and direction of the relationship between L1 RT and the Simon effect. The interaction between Simon effect and Language indicated the relationship between the Simon effect and reversed language domaince effect. The interaction between Simon effect and Trial type estimated the size and direction of the relations between the L1 switching cost and the Simon effect. The three-way interaction between Simon effect, Language, and Trial type estimated whether the Simon effect would have an effect on the asymmetry of switching cost.

\section{Results}

For the cued language-switching task, incorrect RTs $(6.43 \%$, including language errors and naming errors) were first removed. Then, responses following incorrect ones (2.44\%) and responses named with hesitation $(1.25 \%)$ were eliminated. Absolute outliers (below $200 \mathrm{~ms}$ and above 2,000 ms, representing $1.70 \%$ of the data) and relative outliers ( 2.5 standard deviations slower or faster than each participant's mean RT, representing $1.94 \%$ of the data) were excluded. For the Simon task, incorrect RTs $(2.19 \%)$ were first removed, and then relative outliers (2.5 standard deviations slower or faster than each participant's mean RT, representing $3.01 \%$ of the data) were eliminated.

The results of the cued language-switching task are shown in Fig. 1. As shown in Table 2, the language parameter was significantly negative, $t=-5.47, p<0.001$, indicating that the RT of L1 was longer than that of L2, which suggests a global slowing effect in L1. The trial type parameter was significantly positive, $t=8.77, p<0.001$, indicating a significant 

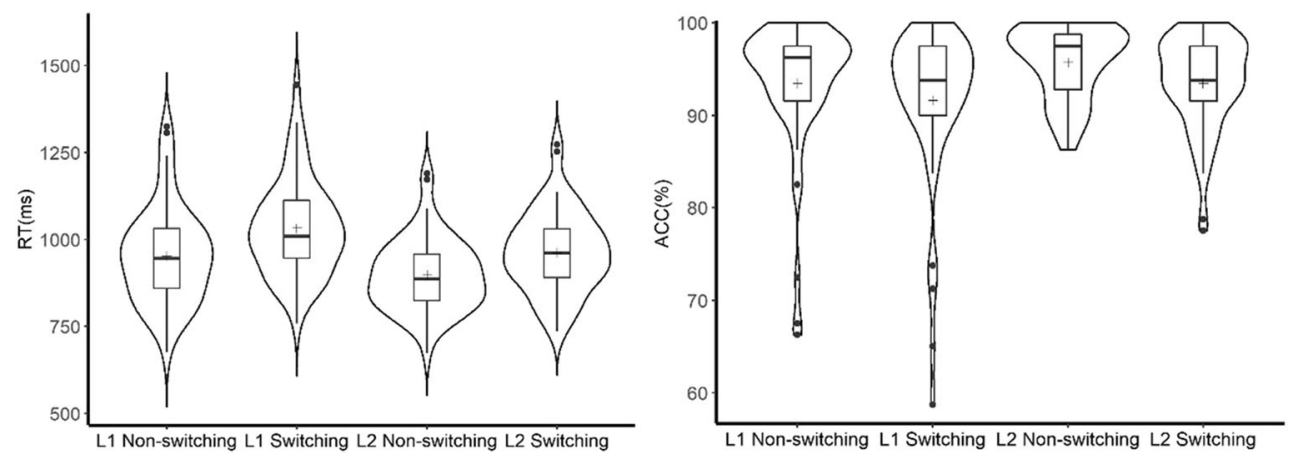

Fig. 1 Mean reaction times (RTs; left panel) and accuracy rate (ACC; right panel) for different conditions in Experiment 1

switching cost in L1. No asymmetrical switching cost was found, because Language $\times$ Trial type parameter was not significant. The Simon effect $\times$ Trial type parameter was significantly negative, $t=-2.69, p=0.010$, indicating that a larger Simon effect was associated with a smaller L1 switching cost. The significant Simon effect $\times$ Language $\times$ Trial type parameter $(t=2.33, p=0.024)$ indicated that the Simon effect predicted the asymmetry of switching costs: the extent to which L1 switch costs were larger than L2 switch costs became smaller as the Simon effect became larger (see Fig. 2). A post hoc power analysis was performed on the significant Simon effect $\times$ Language $\times$ Trial type interaction by Monte Carlo simulation (number of simulation $=1,000$ ) using the simr package in R (Arend \& Schfer, 2018; Green et al., 2016). This analysis yielded an estimated high power $(>80 \%)$ between $99.63 \%$ and $100 \%$ for such an effect (95\% confidence interval (CI)). Especially, we did not find any interaction between language and trial type (namely, no asymmetric switch costs) or significant association between the Simon effect and the reversed-language dominance effect (as seen in Tables 2).

To test the correlation between L2 switching cost and the Simon effect, dummy coding was performed with L2 as the baseline ( $\mathrm{L} 2=0, \mathrm{~L} 1=1)$. As shown in Table 3, a significant L2 switching cost was found, $t=8.34, p<0.001$, but the
Simon effect $\times$ Trial type parameter was not significant, indicating that L2 switching cost was not affected by the Simon effect.

From Fig. 2, it seemed that participants were fairly evenly distributed around a switch cost asymmetry value of zero namely, about half had larger switch costs in L1 and half had larger switch costs in L2. One might doubt that the association we reported above indicated that participants with larger Simon effects (weaker interference inhibition abilities) seemlingly had larger switch costs in L2 than in L1. To test what the pattern of switch costs was in participants with lower interference inhibition ability, we divided these participants into two groups (i.e., high-interference inhibition group vs. low-interference inhibition group) based on the median split method. Then we performed a three-way ANOVA with the within-subject factors language (L1 vs. L2) and Trial type (switch vs. nonswitch) and the between-subject factor Group (high-interference inhibition group vs. low-interference inhibition group). The three-way interaction was significant, $F(1,52)=5.22, p<0.05, \eta^{2}=0.09$. A further paired-samples t-test showed that L1 switch costs did not differ from L2 switch costs for the low-interference inhibition group, $t(26)<$ 1; but L1 switch costs were larger than L2 switch costs for the high-interference inhibition group, $t(26)=2.31, p<0.05$.

Table 2 Mixed-effects model for response time (RT) in Experiment $1(\mathrm{~L} 1=$ baseline)

\begin{tabular}{lcrrr}
\hline Fixed effects & Estimate & $S E$ & $t$-ratio & $p$-value \\
\hline Intercept & 993.98 & 20.50 & 48.49 & $<0.001$ \\
Language & -59.93 & 10.96 & -5.47 & $<0.001$ \\
Trial type & 78.89 & 8.99 & $<.77$ & -1.02 \\
Language $\times$ Trial type & -12.18 & 11.96 & 0.14 \\
Simon effect & 2.71 & 18.81 & -0.60 & 0.314 \\
Simon effect $\times$ Language & -4.70 & 7.79 & -2.69 & 0.886 \\
Simon effect $\times$ Trial type & -19.86 & 7.38 & 2.33 \\
Simon effect $\times$ Language $\times$ Trial type & 23.14 & 9.95 & 0.010 \\
\hline
\end{tabular}

$S E$ standard error

Model formula: RT $=$ Simon effect $\times$ Language $\times$ Trial type $+($ Language $\times$ Trial type $+1 \mid$ subject $)+($ Language $\times$ Trial type $+1 \mid$ item $)$ 


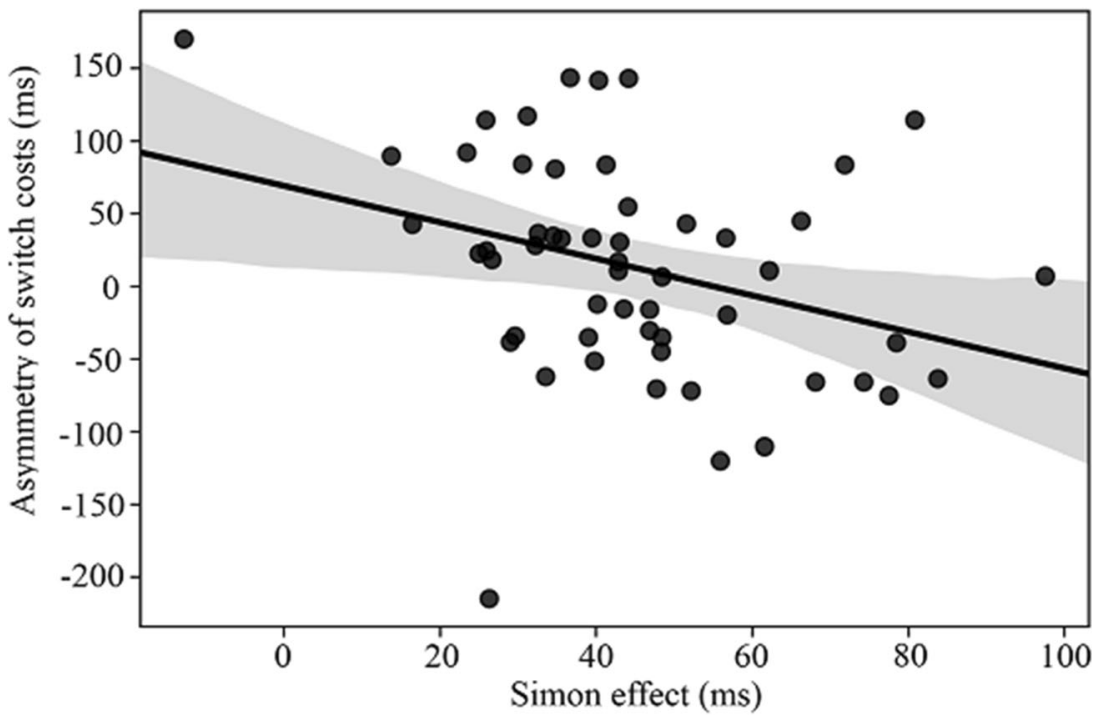

Fig. 2 Relationship between Simon effect and asymmetry of switching costs (L1 switching cost - L2 switching cost) in Experiment 1

\section{Discussion}

Experiment 1 examined the effects of interference suppression on the language control mechanism at different levels in bilinguals.

Consistent with previous studies (e.g., Costa \& Santesteban, 2004; Ma et al., 2016; Meuter \& Allport, 1999), we found significant switching costs for both languages. That is, the RT for switching trials was longer than that for non-switching trials in both L1 and L2. According to the IC model, bilinguals need to inhibit the non-target language when they switch between two languages. Switching costs are generated when overcoming inhibition that lasts from the previous trial to the current one.

It was also found that the Simon effect significantly predicted the asymmetry of switching costs although the results did not show an overall asymmetry in switch costs. In previous studies, the Simon effect was often used as a measure of interference suppression, such that a smaller Simon effect indicated better interference inhibition ability (e.g., Bialystok et al., 2004; Grady et al., 2015; Simon \& Rudell, 1967). It is believed that the asymmetry of switching costs indicates bilingual language control at the local level (e.g., Guo, Liu, Misra, \& Kroll, 2011; Ma et al., 2016; Wu et al., 2018; for review, see Declerck, 2020). When participants with better interference inhibition ability name in L2, they automatically exert more inhibition on L1, so more resources are needed to remove inhibition when switching from L2 to L1, resulting in a larger switching cost for L1 (i.e., asymmetrical switching costs). Participants with weaker interference inhibition ability exert less inhibition and produce symmetric switching costs. However, this result was inconsistent with the findings of Liu et al. (2014). We believe that this may be due to the loss of information in their study caused by converting continuous predictors into categorical factors. In the study of Liu et al. (2018), the Stroop effect was used to divide participants into

Table 3 Mixed-effects model for response time (RT) in Experiment $1(\mathrm{~L} 2=$ baseline)

\begin{tabular}{|c|c|c|c|c|}
\hline Fixed effects & Estimate & $S E$ & $t$-ratio & $p$-value \\
\hline Intercept & 934.04 & 17.54 & 53.24 & $<0.001$ \\
\hline Language & 59.94 & 10.95 & 5.47 & $<0.001$ \\
\hline Trial type & 66.71 & 8.00 & 8.34 & $<0.001$ \\
\hline Language $\times$ Trial type & 12.18 & 11.96 & 1.02 & 0.314 \\
\hline Simon effect & -1.98 & 14.93 & -0.13 & 0.895 \\
\hline Simon effect $\times$ Language & 4.69 & 7.79 & 0.60 & 0.550 \\
\hline Simon effect $\times$ Trial type & 3.28 & 7.45 & 0.44 & 0.661 \\
\hline Simon effect $\times$ Language $\times$ Trial type & -23.15 & 9.95 & -2.33 & 0.024 \\
\hline
\end{tabular}

$S E$ standard error

Model formula: RT $=$ Simon effect $\times$ Language $\times$ Trial type $+($ Language $\times$ Trial type $+1 \mid$ subject $)+($ Language $\times$ Trial type $+1 \mid$ item $)$. 
high- and low-inhibition groups, but no effect of inhibition on the asymmetry of switching costs was found. In the present study, interference suppression was put into a mixed-effects model as a continuous variable, taking into account the differences in subjects and items. Therefore, the results of this experiment can be generalized to other groups of subjects and items.

In line with the study of De Bruin and her colleagues (de Bruin et al., 2014), we found a negative correlation between L1 switching cost and the Simon effect, while Linck et al. (2012) showed the opposite pattern. Theses contradictory results may be due to the different repetition times of each picture. Bexkens, van den Wildenberg, and Tijms (2014) reported that the Simon effect was a predictor of rapid automatized naming (RAN) where each stimulus was repeated several times in dyslexic children. They believed that inhibition was needed to resolve the competition between previously activated stimuli and the current stimulus, which had been caused by RAN. Each picture was repeated eight times in the current experiment and 15 times in the mixed block in the De Bruin et al. (2014) study, but 60 times in the Linck et al. (2012) study. Jylkkä et al. (2017) believed that the frequent repetition times of stimulus may lead to the maintenance of the picturelanguage pairings in working memory, possibly interfering with the cued language-switching task, and more inhibition was needed to resolve this interference. Therefore, the number of picture repetitions might cause different association patterns with interference suppression.

In the present study, it was also found that unbalanced bilinguals were slower when naming pictures in L1 relative to $\mathrm{L} 2$, indicating a reversed language domaince effect. The result is consistent with previous studies (e.g., Costa \& Santesteban, 2004; Gollan et al., 2014; Gollan \& Ferreira, 2009; Kleinman \& Gollan, 2018; Misra, Guo, Bobb, \& Kroll, 2012). Compared with switching costs, the reversed language dominance effect may reflect the global level of language control in mixed language context, i.e. stronger inhibition of the dominant language in order to prevent conflicts (e.g., Bobb \& Wodniecka, 2013; Kleinman \& Gollan, 2018; Kroll et al., 2006; Wu et al., 2018).

However, the results of Experiment 1 did not show any correlation between the reversed language dominance effect and the Simon effect. One possible reason is that the language control mechanism may be affected by different components of IC at different levels. The asymmetry of switching costs and the Simon effect both emphasize the inhibition of stimuli, which occurs between trials, while the reversed language dominance effect emphasizes the global inhibition of responses. Bialystok et al. (2006) argued that bilingualism may affect different components of executive function. Interference suppression refers to the inhibition of irrelevant mental representation, and is involved in the process of inhibiting the representation of non-target language in bilinguals. In contrast, response inhibition refers to the ability to inhibit dominant responses, and is involved in the process of inhibiting the non-target language response in bilinguals. Notably, the term interference suppression used in the present study has the same definition as the term $I C$ mentioned by Bialystok et al. Therefore, the language control mechanism at different levels may be affected by different components of IC.

Experiment 2 further examined the effects of response inhibition on the bilingual language control mechanism at different levels. Response inhibition was measured by the go/nogo task, and language control was measured by the same language-switching paradigm employed in Experiment 1.

\section{Experiment 2}

\section{Methods}

\section{Participants}

Another 54 Chinese-English bilinguals (32 females) with an average age of 22.85 years $(S D=1.99)$ participated in Experiment 2. Similarly, we performed a post hoc power analysis based on Monte Carlo simulation (nsim = 1,000) using the simr package in R (Arend \& Schfer, 2018; Green et al., 2016). All of them were right-handed with normal or corrected vision, and had not participated in Experiment 1. They began learning English at an average age of 9.44 years $(S D=2.38)$. All participants passed the CET-4 with an average score of $526(S D=51)$. A paired-sample $t$-test performed on their self-rating scores showed that their proficiency in Chinese was significantly higher than that in English, $t(53)=$ $14.42, p<0.001$, indicating that the participants were unbalanced bilinguals. Detailed information about participants' language and cognitive abilities is shown in Table 1. The participants who completed Experiment 2 were similar with those of Experiment 1, with no difference in language proficiency, age of L2 acquisition, working memory, or fluid intelligence (see Table 1).

\section{Materials}

The same materials as in Experiment 1 were used.

\section{Procedure}

The same procedure as in Experiment 1 was employed, the only difference being that all participants were required to complete a go/no-go task instead of the Simon task. In the go/no-go task, for each trial, a fixation cross was displayed for $350 \mathrm{~ms}$ in the center of the screen, followed by a blank screen for $150 \mathrm{~ms}$. Then, an experimental stimulus was 
presented for 2,000 ms. To reduce the stimulus repetition effect, there were four different stimuli including two types of shapes (squares or triangles) in two colors (red or blue). The squares and triangles were set to appear at a ratio of $4: 1$. Participants were required to react to squares (go condition) rather than triangles (no-go condition). For red squares, the BACKSLASH key at the right side of the keyboard was pressed, and for blue squares, the TAB key at the left side of the keyboard was pressed. The formal experiment consisted of 750 trials, which were divided into three blocks. The average correct-response RT to go trials was taken as a measure of the response suppression ability.

\section{Data analysis}

Correct-response latencies were analyzed using linear mixedeffects models with random effects of participants and items. Mixed-effects models were fitted using the lme4 package version 1.1-21 (Bates et al., 2014) and the lmerTest package version 3.1-0 (Kuznetsova et al., 2015) in the R language environment (R Development Core Team, 2016).

As in Experiment 1, RTs for go trials were centred around the mean value to help reduce collinearity within the model (Cunnings, 2012). In this model, Language (L1, L2), trial type (switching, non-switching), and centered RT in go trials were fixed effects. The model also included random intercepts for subjects and items, and by-subject and by-item random slopes for language, trial type, and their interaction. Significance ( $p$ value) was calculated with Satterthwaite's approximations (Kuznetsova et al., 2015). Dummy coding was performed for language trials, with $\mathrm{L} 1$ as the baseline $(\mathrm{L} 1=0, \mathrm{~L} 2=1)$; contrast coding was performed for trial-type trials, with nonswitching as the baseline (non-switching $=-0.5$, switching $=$ $0.5)$.

\section{Results}

For the cued language-switching task, incorrect RTs $(8.77 \%$, including language errors and naming errors) were first removed. Then, responses following the incorrect ones $(3.13 \%)$ and responses given with hesitation $(1.46 \%)$ were eliminated. Absolute outliers (below $200 \mathrm{~ms}$ and above $2,000 \mathrm{~ms}$, representing $5.37 \%$ of the data) and relative outliers (2.5 standard deviations slower or faster than each participant's mean RT, representing $1.85 \%$ of the data) were excluded. For the golno-go task, incorrect go trials $(2.90 \%)$ and nogo trials $(2.52 \%)$ were first removed, and then relative outliers (2.5 standard deviations slower or faster than each participant's mean RT, representing $2.41 \%$ of the data) were eliminated. Speed-accuracy tradeoff analyses revealed no significant relation between $\mathrm{d}^{\prime}\left(\mathrm{d}^{\prime}=\mathrm{z}\right.$ (correct go trials rate) $-\mathrm{z}$ (error nogo trials rate), Schulz et al., 2007) and RT $\left(r_{(52)}=0.06 ; p=\right.$ 0.683 ).

The results of the language-switching task are shown in Fig. 3. As shown in Table 4, the language parameter was significantly negative, $t=-4.33, p<0.001$, indicating a global slowing effect. The trial-type parameter was significantly positive, $t=7.56, p<0.001$, indicating a significant $\mathrm{L} 1$ switching cost. The RT go trials by language parameter interaction was significantly positive, $t=2.30, p=0.026$, which indicated a relationship between RT in go trials and the global slowing effect (see Fig. 4). A post hoc power analysis was performed on the significant RT go trials by language parameter interaction by Monte Carlo simulation (number of simulation = 1,000 ) using the simr package in R (Arend \& Schfer, 2018; Green et al., 2016). This analysis yielded an high estimated power (> 80\%) between $99.63 \%$ and $100 \%$ for such an effect (95\% CI).

To test the relations between $\mathrm{L} 2$ switching cost and RT in go trials, dummy coding was performed with L2 as the baseline $(\mathrm{L} 2=0, \mathrm{~L} 1=1)$. As shown in Table 5 , a significant $\mathrm{L} 2$ switching cost was found, $t=10.15, p<0.001$. Notably, these results indicated that there was no interaction between language and trial type (namely, no asymmetry), and that go RTs were not associated with the magnitude of switch costs in either language (as seen in Tables 4 and 5).

\section{Discussion}

Experiment 2 examined the impact of response inhibition on the bilingual language control mechanism at different levels. The participants were comparable to those of Experiment 1, with no difference in language proficiency, age of L2 acquisition, working memory, or fluid intelligence.

Table 4 Mixed-effects model for response time (RT) in Experiment 2 $(\mathrm{L} 1=$ baseline $)$

\begin{tabular}{lrrrrr}
\hline Fixed effects & Estimate & $S E$ & $t$-ratio & $p$-value \\
\hline Intercept & 1065.47 & 20.87 & 51.04 & $<0.001$ \\
Language & -52.40 & 12.10 & -4.33 & $<0.001$ \\
Trial type & 76.73 & 10.16 & 7.56 & $<0.001$ \\
Language $\times$ Trial type & 1.70 & 11.28 & 0.15 & 0.881 \\
RT go trials & 61.53 & 18.84 & 3.27 & 0.002 \\
RT go trials $\times$ Language & 18.54 & 8.06 & 2.30 & 0.026 \\
RT go trials $\times$ Trial type & -6.86 & 7.84 & -0.88 & 0.386 \\
RT go trials $\times$ Language $\times$ Trial type & 9.55 & 9.05 & 1.06 & 0.296 \\
\hline
\end{tabular}

$S E$ standard error

Model formula: $\mathrm{RT}=\mathrm{RT}$ go trials $\times$ Language $\times$ Trial type $+($ Language $\times$ Trial type $+1 \mid$ subject $)+($ Language $\times$ Trial type $+1 \mid$ item $)$ 



Fig. 3 Mean response times (RTs; left panel) and accuracy rate (ACC; right panel) for different conditions in Experiment 2

Similar to Experiment 1, Experiment 2 showed significant switching costs for both languages and the reversed language dominance effect, but no overall switch cost asymmetry. This further indicated that bilinguals in the two experiments were similar, and that the results in the two experiments were consistent and reliable.

Interestingly, Experiment 2 also showed that RTs for go trials significantly predicted the reversed language dominance effect. Specifically, faster RTs for go trials were associated with larger reversed language dominance effects. In previous studies, RT to go trials was often used as a measure for response inhibition, and faster RTs to go trials were considered an index of better response inhibition (e.g., Benikos et al., 2013; Jodo \& Inoue, 1990; Manuel et al., 2010). The reversed language dominance effect represents stronger inhibition of L1 at the global level (e.g., Bobb \& Wodniecka, 2013; Kleinman \& Gollan, 2018; Kroll et al., 2006; Wu et al., 2018). Although no studies have been carried out to directly examine the effect of response inhibition (as measured in the go/no-go task) on language control, some studies have found that bilinguals showed advantages in tasks associated with response inhibition. For example, Fernandez, Tartar, Padron, and Acosta (2013) and Moreno, Wodniecka, Tays, Alain, and Bialystok (2014) found that under no-go conditions, bilinguals produced a more negative N2 event-related potential response than monolinguals, indicating that bilinguals had better response-inhibition ability. In addition to these crosssectional studies, the longitudinal study of Sullivan, Janus, Moreno, Astheimer, and Bialystok (2014) examined whether short-term second language learning could have an early impact on executive control. The training group was given 6 months of introductory Spanish learning, while the control group was given 6 months of introductory psychology learning. The results showed that compared with the pre-test, the P3 amplitude increased in the training group after the go/nogo task, while the control group showed no such change, indicating that second-language learning improved the executive control ability of participants. It is believed that bilinguals inhibit the non-target language and dominant responses in similar ways, so bilinguals perform better in responseinhibition tasks than monolinguals (e.g., Fernandez et al., 2013; Moreno et al., 2014; Sullivan et al., 2014; but see, e.g., Paap \& Greenberg, 2013). The current results confirmed previous findings from another perspective.

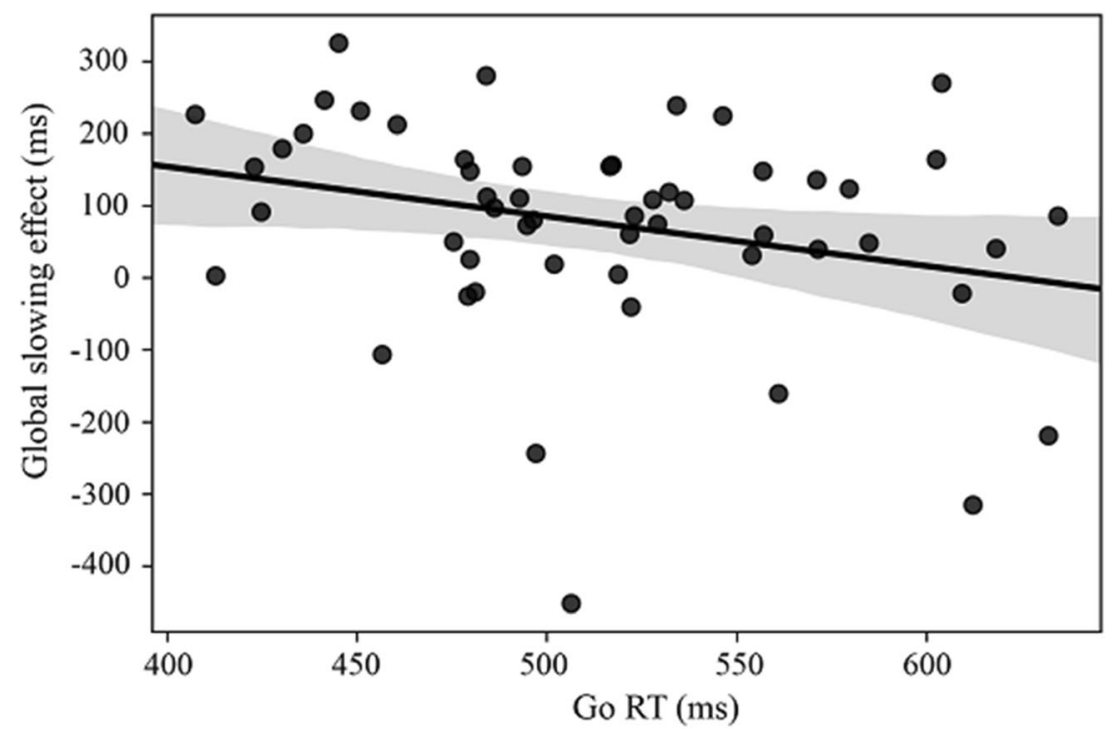

Fig. 4 Relationship between go respone times (RTs) and global slowing effect (L1 RT - L2 RT) in Experiment 2 
Table 5 Mixed-effects model for response time (RT) in Experiment 2 (L2 = baseline)

\begin{tabular}{lrrrrr}
\hline Fixed effects & Estimate & $S E$ & $t$-ratio & $p$-value \\
\hline Intercept & 1013.08 & 22.29 & 45.45 & $<0.001$ \\
Language & 52.40 & 12.10 & 4.33 & $<0.001$ \\
Trial type & 78.43 & 7.73 & 10.15 & $<0.001$ \\
Language $\times$ Trial type & -1.70 & 11.28 & -0.15 & 0.881 \\
RT go trials & 80.07 & 20.06 & 3.99 & $<0.001$ \\
RT go trials $\times$ Language & -18.54 & 8.06 & -2.30 & 0.026 \\
RT go trials $\times$ Trial type & 2.69 & 7.15 & 0.38 & 0.708 \\
RT go trials $\times$ Language $\times$ Trial type & -9.55 & 9.05 & -1.06 & 0.296 \\
\hline
\end{tabular}

$S E$ standard error

Model formula: RT $=$ RT go trials $\times$ Language $\times$ Trial type $+($ Language $\times$ Trial type $+1 \mid$ subject $)+($ Language $\times$ Trial type $+1 \mid$ item $)$

However, Experiment 2 did not show any correlation between the asymmetry of switching costs and response inhibition (i.e., the interaction between RTs to go trials and trial type was not significant), which was consistent with the findings of De Bruin et al. (2014). Bialystok et al. (2006) argued that responses in the nontarget language must be inhibited for bilinguals, which would enhance their response-inhibition ability, but the inhibition of the representation of nontarget language would not.

\section{General discussion}

Across two experiments, we examined how two distinct components of IC (i.e., interference suppression and response inhibition) affect language control at the local and global levels. Experiment 1 revealed that bilinguals with stronger interference suppression ability had stronger local language control. Experiment 2 revealed that bilinguals with stronger responseinhibition ability had better global language control. Overall, these results indicate that different components of IC are associated with distinct levels of language control in bilinguals.

First, Experiment 1 revealed that the Simon effect significantly predicted the asymmetry of switching costs although niether experiment showed an overall asymmetry in switch costs. In fact, the findings of asymmetric switching costs in previous studies have not always been consistent. Meuter and Allport (1999) were among the first to reveal the asymmetry of switching costs in unbalanced bilinguals with a dominant L1. Since then, several studies replicated this finding in unbalanced bilinguals (e.g., Costa \& Santesteban, 2004, Experiment 1; Ma et al., 2016, Experiment 1; Philipp et al., 2007, Experiment 1). However, some other studies reported symmetric switching costs (e.g., Costa \& Santesteban, 2004, Experiments 2-5; Finkbeiner et al., 2006; Gollan \& Ferreira,
2009; Gollan et al., 2014; Verhoef et al., 2009), suggesting that some factors may modulate the asymmetry of switching costs. For example, Costa and Santesteban (2004) found symmetric switching costs when trilinguals switched between their two dominant languages (Experiments 2 and 3) or between their stronger language (L1) and their much weaker language (L3; Experiment 4). These authors argued that L2 proficiency or L2 age of acquisition may influence languageswitching performance. However, a later study ruled out the possibility of the influence of L2 age of acquisition on symmetric switching costs, and confirmed the influence of language proficiency (Costa, Santesteban, \& Ivanova, 2006). Based on the results of the current study, bilinguals' interference suppression ability may be another influential factor that modulates the asymmetry of switching costs. More specifically, bilinguals with lower interference suppression ability may show symmetric switching costs, while those with higher interference suppression ability may show asymmetric switching costs. In contrast, the current results did not provide any evidence for the relationship between response-inhibition ability and asymmetry of switching costs. Future studies may go further to investigate how theses factors interact with each other, and to investigate this issue by using other tasks such as the stop-signal task.

Furthermore, we found the reversed language domiance effect in both experiments, in line with many previous studies (e.g., Christoffels et al., 2007; Costa \& Santesteban, 2004; Gollan \& Ferreira, 2009; Misra et al., 2012; Verhoef et al., 2009). Some studies still observed such a reversed language dominance effect, even when there was no evidence for the asymmetry of switching costs (e.g., Costa \& Santesteban, 2004, Experiments 2-5; Gollan \& Ferreira, 2009). Like the asymmetry of switching costs, the reversed-language dominance effect is also affected by some factors such as relative accessibility of the two languages (e.g., Declerck et al., 2020) and types of stimuli (e.g., Meuter \& Allport, 1999). The current study extended the previous findings by showing that bilinguals' response-inhibition ability may be related to the reversed-language dominance effect. That is, bilinguals with better response-inhibition ability show a larger reversedlanguage dominance effect, while those with lower response-inhibition ability may show a smaller reversedlanguage dominance effect.

By using the asymmetry of language switching costs as an index for reactive control at the local level, and the reversed language domiance effect as an index for proactive control at the global level, the present study provides further evidence that these two levels of language control are recruited to support lexical selection during language production. According to the IC model (Green, 1998), bilinguals inhibit the nontarget language reactively in nature (De Groot, 1998; De Groot \& Christoffels, 2006), and reactive control refers to the inhibition after the activation (Green, 1998). More recent 
studies have suggested the possibility of two types of language control. That is, apart from reactive control, proactive control also modulates activation of language nodes of these two languages before their lexical activation (e.g., De Groot \& Christoffels, 2006; Kleinman \& Gollan, 2018; Van Assche, Duyck, \& Gollan, 2013; Wu et al., 2018). The results of the current study are consistent with the assumption that language control is exerted by two complementary processes: a global and proactive process that increases activation of lemmas in the target language while decreasing activation of lemmas the non-target language and a local and reactive process that suppresses activation of lemmas in the non-target language that might have escaped the global inhibition process (Van Assche et al., 2013).

In addition, the current findings also reveal that domain-general IC is highly related to different levels of language control. As one of the important components of executive function, IC refers to the ability to inhibit inappropriate responses to achieve a desired target (Aron, Robbins, \& Poldrack, 2004). It consists of at least two sub-components: response inhibition and interference suppression (Nigg, 2000).

Previous studies (e.g., Linck et al., 2012; Liu et al., 2014; Liu, Liang, et al., 2016b) have shown that domain-general IC ability plays a role in language control such that individuals with better IC also have a better local language control performance. For example, Linck et al. (2012) found that the Simon effect correlates positively with the L1 switching cost, indicating that the smaller the Simon effect (i.e., stronger IC ability), the smaller the L1 switching cost (i.e., better language switching capability). Similarly, Liu et al. (2014) divided bilinguals into two groups based on their IC as measured with the Simon arrow task. It was found that bilinguals with lower IC ability had asymmetrical switching costs and bilinguals with higher IC ability had symmetrical switching costs.

The present study found that different components of IC ability are associated with different levels of language control. Specifically, bilinguals' interference suppression ability is correlated with local language control, whereas their response-inhibition ability is related to global language control. This is the first study that systematically examined how different components of domain-general IC influence different levels of language control. The results not only support the proposal that generic IC is highly correlated with language control in bilinguals, but also reveal specific relationships between two different components of IC and two different levels of language control. On the other hand, diverging results have been shown in previous studies on bilingual advantage in cognitive control. Some studies have found that bilingual language experience has a significant impact on interference suppression or response inhibition (e.g., Bialystok et al., 2004; Fernandez et al., 2013), while some other studies have failed to observe an explicit cognitive benefit in bilinguals (Dick et al., 2019; Paap \& Greenberg, 2013; Von Bastian, Souza, $\&$ Gade, 2016). One possible reason is that different types of language experience might influence different components of cognitive control tapped by different tasks (for a similar line of argumentation, see Poarch \& Krott, 2019). From this perspective, the current findings also have important implications for future studies to take these factors into account. Finally, it should be noted that, although the two groups of participants in two experiments were well matched in age, L2 AoA, CETscores, L1 self-rating scores, L2 self-rating scores, O-span scores, and Raven scores, it would be more reasonable to ask one group of participants to complete the Simon task, the go/no-go task, and the cued picture-naming task in order to exclude any potential possibility of individual differences.

Acknowledgements The study was supported by the National Natural Science Foundation of China [31871097], the National Key Basic Research Program of China [2014CB846102], the Interdiscipline Research Funds of Beijing Normal University, and the Fundamental Research Funds for the Central Universities [2017XTCX04]. The authors declared no conflict of interest.

All data in this manuscript have been made available on Mendeley Data at: https://data.mendeley.com/datasets/f4c69tn945/draft?a= d95d4958-51fe-4293-8ca0-bc6b39de3a04. The study has not been preregistered.

\section{References}

Arend, M. G., \& Schfer, T. (2018). Statistical power in two-level models: a tutorial based on monte carlo simulation. Psychological Methods, 24(1), 1-19.

Aron, A. R., Robbins, T. W., \& Poldrack, R. A. (2004). Inhibition and the right inferior frontal cortex. Trends in cognitive sciences, 8(4), 170177.

Bates, D., Maechler, M., Bolker, B., \& Walker, S. (2014). lme4: Linear mixed-effects models using Eigen and S4. R package version, 1(7), $1-23$.

Benikos, N., Johnstone, S. J., \& Roodenrys, S. J. (2013). Short-term training in the Go/Nogo task: behavioural and neural changes depend on task demands. International Journal of Psychophysiology, 87(3), 301-312.

Bexkens, A., van den Wildenberg, W. P. M., \& Tijms, J. (2014). Rapid automatized naming in children with dyslexia: Is IC involved? Dyslexia, 21, 212-234.

Bialystok, E., Craik, F. I., Klein, R., \& Viswanathan, M. (2004). Bilingualism, aging, and cognitive control: evidence from the Simon task. Psychology and aging, 19(2), 290-303.

Bialystok, E., Craik, F. I., \& Ryan, J. (2006). Executive control in a modified antisaccade task: effects of aging and bilingualism. Journal of Experimental Psychology Learning Memory \& Cognition, 32(6), 1341-1354.

Bobb, S. C., \& Wodniecka, Z. (2013). Language switching in picture naming: what asymmetric switch costs (do not) tell us about inhibition in bilingual speech planning. Journal of Cognitive Psychology, 25(5), 568-585.

Bunge, S. A., Dudukovic, N. M., Thomason, M. E., Vaidya, C. J., \& Gabrieli, J. D. (2002). Immature frontal lobe contributions to cognitive control in children: Evidence from fMRI. Neuron, 33(2), 301311. 
Calabria, M., Hernández, M., Branzi, F. M., \& Costa, A. (2012) Qualitative differences between bilingual language control and executive control: Evidence from task-switching. Frontiers in Psychology, doi: https://doi.org/10.3389/fpsyg.2011.00399. Published online by Frontiers, January 13, 2012.

Chen, M., Wu, Y. J., Wu, J., Fu, Y., Li, S., Liu, H., Lu, C., \& Guo, T. (2019). Individual differences in IC abilities modulate the functional neuroplasticity of IC. Brain Structure \& Function, 224, 2357-2371.

Christoffels, I. K., Firk, C., \& Schiller, N. O. (2007). Bilingual language control: an event-related brain potential study. Brain Research, $1147,192-208$

Costa, A., \& Caramazza, A. (1999). Is lexical selection in bilingual speech production language-specific? Further evidence from Spanish-English and English-Spanish bilinguals. Bilingualism: Language and Cognition, 2(3), 231-244.

Costa, A., \& Santesteban, M. (2004). Lexical access in bilingual speech production: evidence from language switching in highly proficient bilinguals and 12 learners. Journal of Memory \& Language, 50(4), 491-511.

Costa, A., Santesteban, M., \& Ivanova, I. (2006). How do highly proficient bilinguals control their lexicalization process? Inhibitory and language-specific selection mechanisms are both functional. Journal of Experimental Psychology: Learning, Memory and Cognition, 32(5), 1057-1074.

Cunnings, I. (2012). An overview of mixed-effects statistical models for second language researchers. Second Language Research, 28(3), 369-382.

de Bruin, A., Roelofs, A., Dijkstra, T., \& Fitzpatrick, I. (2014). Domaingeneral inhibition areas of the brain are involved in language switching: fMRI evidence from trilingual speakers. Neuroimage, 90, 348-359.

Declerck, M., Grainger, J., Koch, I., \& Philipp, A.M. (2017). Is language control just a form of executive control? Evidence for overlapping processes in language switching and task switching. Journal of Memory and Language, 95, 138-145.

Declerck, M. (2020). What about proactive language control? Psychonomic Bulletin \& Review, 27, 24-35.

Declerck, M., Kleinman, D., \& Gollan, T. H. (2020). Which bilinguals reverse language dominance and why?. Cognition, 204, 104384.

De Groot, A. M. B. (1998). Retroactive or proactive control of the bilingual system. Bilingualism: Language and Cognition, 1(2), 86-87.

De Groot, A. M. B., \& Christoffels, I. K. (2006). Language control in bilinguals: Monolingual tasks and simultaneous interpreting. Bilingualism: Language and Cognition, 9(02), 189-201.

Dick, A. S., Garcia, N. L., Pruden, S. M., Thompson, W. K., Hawes, S. W., Sutherland, M. T., ... Gonzalez, R. (2019). No evidence for a bilingual executive function advantage in the ABCD study. Nature Human Behaviour, 3(7), 692-701.

Fernandez, M., Tartar, J. L., Padron, D., \& Acosta, J. (2013). Neurophysiological marker of inhibition distinguishes language groups on a non-linguistic executive function test. Brain and Cognition, 83(3), 330-336.

Finkbeiner, M., Almeida, J., Janssen, N., \& Caramazza, A. (2006). Lexical selection in bilingual speech production does not involve language suppression. Journal of Experimental Psychology: Learning, Memory, and Cognition, 32, 1075-1089.

Friedman, N. P., \& Miyake, A. (2004). The relations among inhibition and interference control functions: a latent-variable analysis. Journal of Experimental Psychology: General, 133(1), 101-135.

Fu, Y., Lu, D., Kang, C., Wu, J., Ma, F., Ding, G., \& Guo, T. (2017). Neural correlates for naming disadvantage of the dominant language in bilingual word production. Brain and Language, 175, 123-129.

Gollan, T. H., \& Ferreira, V. S. (2009). Should i stay or should i switch? A cost-benefit analysis of voluntary language switching in young and aging bilinguals. Journal of Experimental Psychology: Learning Memory and Cognition, 35(3), 640-665.
Gollan, T. H., Kleinman, D., \& Wierenga, C. E. (2014). What's easier: doing what you want, or being told what to do? Cued versus voluntary language and task switching. Journal of Experimental Psychology: General, 143(6), 2167-2195.

Grady, C. L., Luk, G., Craik, F. I., \& Bialystok, E. (2015). Brain network activity in monolingual and bilingual older adults. Neuropsychologia, 66, 170-181.

Green, D. W. (1998). Mental control of the bilingual lexico-semantic system. Bilingualism Language \& Cognition, 1(2), 67-81.

Green, P., Macleod, C. J., \& Nakagawa, S. (2016). Simr: an r package for power analysis of generalized linear mixed models by simulation. Methods in Ecology \& Evolution, 493-498.

Guo, T., Liu, H., Misra, M., \& Kroll, J. F. (2011). Local and global inhibition in bilingual word production: fMRI evidence from Chinese-English bilinguals. NeuroImage, 56(4), 2300-2309.

Guo, T., \& Peng, D. (2006). Event-related potential evidence for parallel activation of two languages in bilingual speech production. Neuroreport, 17(17), 1757-1760.

Jodo, E., \& Inoue, K. (1990). Effects of practice on the P300 in a Go/ NoGo task. Electroencephalography and Clinical Neurophysiology, 76(3), 249-257.

Jylkkä, J., Lehtonen, M., Lindholm, F., Kuusakoski, A., \& Laine, M. (2017). The relationship between general executive functions and bilingual switching and monitoring in language production. Bilingualism: Language and Cognition, 21(3), 505-522.

Kang, C., Ma, F., Li, S., Kroll, J. F., \& Guo, T. (2020). Domain-general inhibition ability predicts the intensity of inhibition on non-target language in bilingual word production: An ERP study. Bilingualism: Language and Cognition, 1-14. https://doi.org/10. 1017/S1366728920000085.

Kleinman, D., \& Gollan, T. (2018). Inhibition Accumulates Over Time at Multiple Processing Levels in Bilingual Language Control. Cognition, 173, 115-132.

Kroll, J. F., Bobb, S. C., \& Wodniecka, Z. (2006). Language selectivity is the exception, not the rule: arguments against a fixed locus of language selection in bilingual speech. Bilingualism Language \& Cognition, 9(2), 119-135.

Kuznetsova, A., Brockhoff, P. B., \& Christensen, R. H. B. (2015). Package 'Imertest'. $R$ package version, $2(0)$.

Linck, J. A., Schwieter, J. W., \& Sunderman, G. (2012). Inhibitory control predicts language switching performance in trilingual speech production. Bilingualism: Language and Cognition, 15(3), 651-662.

Lindqvist, S., \& Thorell, L.B. (2009). A brief report: manipulation task difficulty in laboratory measures of IC. Child Neuropsychology 15(1), 1-7.

Liu, C., Jiao, L., Wang, Z., Wang, M., Wang, R., \& Wu, Y. J. (2018). Symmetries of bilingual language switch costs in conflicting versus non-conflicting contexts. Bilingualism: Language and Cognition, 22(3), 624-636.

Liu, H., Fan, N., Rossi, S., Yao, P., \& Chen, B. (2016a). The effect of cognitive flexibility on task switching and language switching. International Journal of Bilingualism, 20, 563-579.

Liu, H., Liang, L., Dunlap, S., Fan, N., \& Chen, B. (2016b). The effect of domain-general inhibition-related training on language switching: An ERP study. Cognition, 146, 264-276.

Liu, H., Rossi, S., Zhou, H., \& Chen, B. (2014). Electrophysiological evidence for domain-general IC during bilingual language switching. PloS one, 9(10), e110887.

Ma, F., Li, S., \& Guo, T. (2016). Reactive and proactive control in bilingual word production: An investigation of influential factors. Journal of memory and language, 86, 35-59.

Manuel, A. L., Grivel, J., Bernasconi, F., Murray, M. M., \& Spierer, L. (2010). Brain dynamics underlying training-induced improvement in suppressing inappropriate action. Journal of Neuroscience, 30(41), 13670-13678. 
Meuter, R. F. I., \& Allport, A. (1999). Bilingual language switching in naming: Asymmetrical costs of language selection. Journal of Memory and Language, 40(1), 25-40.

Mishra, R. K., \& Singh, N. (2016). The influence of second language proficiency on bilingual parallel language activation in HindiEnglish bilinguals. Journal of Cognitive Psychology, 28(4), 396411.

Misra, M., Guo, T., Bobb, S. C., \& Kroll, J. F. (2012). When bilinguals choose a single word to speak: Electrophysiological evidence for inhibition of the native language. Journal of Memory and Language, 67(1), 224-237.

Moreno, S., Wodniecka, Z., Tays, W., Alain, C., \& Bialystok, E. (2014). Inhibitory control in bilinguals and musicians: event related potential (ERP) evidence for experience-specific effects. PloS one, 9(4), e94169.

Nigg, J. T. (2000). On inhibition/disinhibition in developmental psychopathology: views from cognitive and personality psychology and a working inhibition taxonomy. Psychological Bulletin, 126(2), 220246.

Paap, K. R., \& Greenberg, Z. I. (2013). There is no coherent evidence for a bilingual advantage in executive processing. Cognitive Psychology, 66(2), 232-258.

Philipp, A. M., Gade, M., \& Koch, I. (2007). Inhibitory processes in language switching? Evidence from switching language-defined response sets. European Journal of Cognitive Psychology, 19, 395416.

Plonsky, L. (2011). Study quality in SLA: A cumulative and developmental assessment of designs, analyses, reporting practices, and outcomes in quantitative L2 research (Doctoral dissertation, Michigan State University. Second Language Studies).

Poarch, G. J., \& Krott, A. (2019). A Bilingual Advantage? An Appeal for a Change in Perspective and Recommendations for Future Research. Behavioral Sciences, 9(9), 95.

Prior, A., \& Gollan, T. H. (2011). Good language-switchers are good task-switchers: evidence from Spanish-English and MandarinEnglish bilinguals. Journal of the International Neuropsychological Society, 17(4), 682-691.

Prior, A., \& Gollan, T. H. (2013). The elusive link between language control and executive control: A case of limited transfer. Journal of Cognitive Psychology, 25(5), 622-645.

R Development Core Team (2016). R: A language and environment for statistical computing. Vienna, Austria: R Foundation for Statistical Computing. Available from http://www.R-project.org
Schulz, K. P., Fan, J., Magidina, O., Marks, D. J., Hahn, B., \& Halperin, J. M. (2007). Does the emotional go/no-go task really measure behavioral inhibition? convergence with measures on a non-emotional analog. Archives of Clinical Neuropsychology, 2, 151-160.

Simon, J. R., \& Rudell, A. P. (1967). Auditory SR compatibility: the effect of an irrelevant cue on information processing. Journal of applied psychology, 51(3), 300.

Smith, J. L., Johnstone, S. J., \& Barry, R. J. (2006). Effects of prestimulus processing on subsequent events in a warned go/nogo paradigm: response preparation, execution and inhibition. International Journal of Psychophysiology, 61(2), 121-133.

Snodgrass, J. G., \& Vanderwart, M. (1980). A standardized set of 260 pictures: norms for name agreement, image agreement, familiarity, and visual complexity. Journal of experimental psychology: Human learning and memory, 6(2), 174-215.

Sullivan, M. D., Janus, M., Moreno, S., Astheimer, L., \& Bialystok, E. (2014). Early stage second-language learning improves executive control: evidence from ERP. Brain and Language, 139, 84-98.

Van Assche, E., Duyck, W., \& Gollan, T. H. (2013). Whole-language and item-specific control in bilingual language production. Journal of Experimental Psychology: Learning, Memory, and Cognition, 39(6), 1781-1792.

Van Boxtel, G. J., van der Molen, M. W., Jennings, J. R., \& Brunia, C. H. (2001). A psychophysiological analysis of inhibitory motor control in the stop-signal paradigm. Biological psychology, 58(3), 229-262.

Verhoef, K. M. W., Roelofs, A., \& Chwilla, D. J. (2009). Electrophysiological evidence for endogenous control of attention in switching between languages in overt picture naming. Journal of Cognitive Neuroscience, 22(8), 1832-1843.

Von Bastian, C. C., Souza, A. S., \& Gade, M. (2016). No evidence for bilingual cognitive advantages: A test of four hypotheses. Journal of Experimental Psychology: General, 145(2), 246-258.

Wu, J., Kang, C., Ma, F., Gao, X., \& Guo, T. (2018). The influence of short-term language-switching training on the plasticity of the cognitive control mechanism in bilingual word production. Quarterly Journal of Experimental Psychology, 71, 2115-2128.

Publisher's note Springer Nature remains neutral with regard to jurisdictional claims in published maps and institutional affiliations. 